Revista Electrónica Complutense de Investigación en Educación Musical ISSNe: 1698-7454

http://dx.doi.org/10.5209/RECIEM.55936

\title{
El repertorio musical como elemento posibilitador de un enfoque sociocrítico en Educación Primaria. Estudio del currículum impartido en el municipio de Valencia $^{1}$
}

\author{
Pablo Marín Liébana²; Ana María Botella Nicolás ${ }^{3}$
}

Recibido: 26 de abril de 2017 / Aceptado: 6 de octubre de 2017

Resumen. Este estudio constituye una primera aproximación analítica a una investigación más amplia cuyo objetivo es investigar el desarrollo del pensamiento sociocrítico en estudiantes de $5^{\circ}$ y $6^{\circ}$ de Educación Primaria a través de la utilización de un repertorio musical vinculado a contextos social y educativamente relevantes. Para ello, se ha analizado el repertorio musical utilizado y el grado de acuerdo e implementación del enfoque sociocrítico en los centros públicos de la ciudad de Valencia, aplicando un cuestionario a una muestra de $\mathrm{n}=80$ maestros $(\mathrm{N}=92)$. Los resultados muestran un predominio en las aulas de la música clásico-romántica, escolar y folklórico-tradicional. Además, se demuestra que esta selección está condicionada por las representaciones de los maestros sobre la música. Por otra parte, la mayoría de estos coinciden con los principios del enfoque sociocrítico, pero pocos lo aplican.

Palabras clave: educación musical; pedagogía crítica; repertorio musical; Educación Primaria.

\section{[en] The musical repertoire as an element that enables a socio-critical approach in Elementary Education. Study of the taught curriculum in the city of Valencia}

\begin{abstract}
This study is a first look at a broader field that aims to research the development of a sociocritical thinking by the students in years 5 and 6 of elementary education through the use of a musical repertoire linked to social and educationally relevant contexts. For this purpose, an analysis has been made, in state schools of the city of Valencia, of the most used repertoire and the degree of agreement and implementation of the sociocritical approach by teachers. We sent a questionnaire to $n=80$ teachers $(\mathrm{N}=92)$. The results show a predominance of the classic-romantic, school and folk-traditional music in the classrooms. In addition, it is shown that this selection is conditioned by the teachers' representations of music. Moreover, most of the teachers agree with the socio-critical approach, but few of them apply it. Keywords: music education; critical pedagogy; musical repertoire; Elementary Education.
\end{abstract}

Sumario. 1. Introducción. 2. Fundamentación. 3. Metodología. 4. Resultados. 5. Conclusiones. 6. Referencias bibliográficas.

1 Proyecto financiado por el Ministerio de Educación, Cultura y Deporte a través de una ayuda para la Formación de Profesorado Universitario, dentro del Programa Estatal del Talento y su Empleabilidad, en el marco del Plan Estatal de Investigación Científica y Técnica y de Innovación 2013-2016.

2 Máster en Investigación en Didácticas Específicas por la Universitat de València. Personal Investigador en el Departamento de Didáctica de la Expresión Musical, Plástica y Corporal de la Universitat de València. Pablo. Marin-Liebana@uv.es

3 Profesora Contratada Doctora del Departamento de Didáctica de la Expresión Musical, Plástica y Corporal de la Universitat de València. Musicóloga. Ana.Maria.Botella@uv.es 
Cómo citar: Marín, P. y Botella, A. M. (2018). El repertorio musical como elemento posibilitador de un enfoque sociocrítico en Educación Primaria. Estudio del currículum impartido en el municipio de Valencia. Revista Electrónica Complutense de Investigación en Educación Musical, 15, 19-37.

\section{Introducción}

Hoy en día parece indiscutible que el sistema educativo, además de formar en una serie de contenidos disciplinares, tiene también el objetivo de promover un tipo de sociedad fundamentada en los valores democráticos de libertad, igualdad, respeto y tolerancia hacia la diversidad. En esta dirección, la Ley Orgánica 8/2013, de 9 de diciembre, para la Mejora de la Calidad Educativa, prescribe que dicho sistema debe formar "para el ejercicio de la ciudadanía y para la participación activa en la vida económica, social y cultural, con actitud crítica y responsable y con capacidad de adaptación a las situaciones cambiantes de la sociedad del conocimiento" (LOMCE, 2013, p. 97866). Del mismo modo, el Real Decreto 126/2014, de 28 de febrero, por el que se establece el currículo básico de la Educación Primaria, sitúa las Competencias sociales y cívicas como una de las siete que deben orientar dicha etapa educativa.

La consecución de estos fines viene condicionada por la implementación de un modelo educativo que incorpore enfoques didácticos de tipo sociocrítico, de manera que se problematicen las relaciones sociales desde una perspectiva ética y uno de los objetivos sea la emancipación del sujeto. Para ello, es necesaria una función docente crítica y reflexiva que sitúe los contextos próximos como parte de los contenidos curriculares, de modo que los estudiantes desarrollen herramientas para poder analizar críticamente su entorno y actuar en él desde la responsabilidad moral, contribuyendo así a su conformación como sujetos políticos (Martínez, 2015). En esta dirección, se han desarrollado durante la última década diversas propuestas didácticas y líneas de investigación educativa en las distintas áreas de conocimiento.

Sin embargo, la educación musical en Primaria todavía no cuenta en el territorio español con experiencias didácticas que hayan trascendido significativamente a la comunidad científica ni con un consolidado cuerpo de publicaciones relacionadas con el enfoque sociocrítico. Como sostiene Travé (2003), los enfoques predominantes durante las últimas décadas han priorizado un tratamiento práctico y formalista, dejando de lado la dimensión cultural y social del hecho sonoro. No obstante, cada vez existe un mayor interés por esta última (Díaz, 2006), de manera que se empieza a concebir la música como un elemento válido para estimular una conducta prosocial situada histórica y geográficamente (Cabedo, 2014). Es aquí donde se enmarca esta investigación, cuyo objetivo último es el de estudiar las posibilidades didácticas que ofrece el trabajo en el aula de música desde dicho enfoque. En concreto, se centra en la utilización en $5^{\circ}$ y $6^{\circ}$ de Primaria de un repertorio musical vinculado a problemas socialmente relevantes que permita una participación activa, consciente y reflexiva en el aula, así como una apertura de la escuela a las manifestaciones musicales de los estudiantes y de las distintas tradiciones culturales (García, 2010).

Este proyecto de investigación ha sido financiado por el Ministerio de Educación, Cultura y Deporte para ser desarrollado entre 2016 y 2020. El informe que aquí se presenta corresponde a una primera fase de análisis de la realidad educativa que enfoca su atención en el currículum impartido por los maestros de música de $5^{\circ}$ y $6^{\circ}$ 
curso en los centros públicos de Educación Primaria pertenecientes al municipio de Valencia. Su objetivo es triple. Por un lado, recoger y analizar el tipo de repertorio musical que están utilizando dichos maestros. Por el otro, indagar en sus valoraciones e intervenciones didácticas con respecto al enfoque sociocrítico. En tercer lugar, conocer algunas de sus ideas y concepciones sobre la música y establecer relaciones entre estas y los dos objetivos anteriores. Las hipótesis de partida predicen un mayor uso del repertorio clásico y romántico, como consecuencia de unas concepciones vinculadas al paradigma formalista de la música autónoma. Del mismo modo, se prevé poca presencia de prácticas relacionadas con el enfoque sociocrítico.

\section{Fundamentación}

Este estudio se sustenta teóricamente sobre tres ejes fundamentales: las concepciones de los docentes y su influencia en la innovación educativa, los principios de la pedagogía crítica y su concreción en el enfoque sociocrítico, y las aportaciones de la musicología al propio concepto de música y sus repercusiones en la educación musical. Respecto a la primera, el estudio de las ideas de los docentes y la influencia de estas en el currículum impartido se ha convertido en una línea de investigación con identidad propia. En este sentido, se ha concluido que son especialmente relevantes las creencias relativas a la manera en la que los estudiantes aprenden y sus capacidades evolutivas, el rol del maestro y la importancia relativa de los contenidos (Cronin-Jones, 1991). Además, las concepciones de los maestros y los profesores se han identificado como una de las principales dificultades que encuentran tanto las sucesivas reformas como la incorporación a la práctica educativa de los resultados de la investigación en didáctica (Gil-Pérez y Pessoa de Carvalho, 2000).

En el campo de la educación musical, es posible distinguir dos tipos de investigaciones: aquellas dirigidas a estudiantes universitarios y aquellas cuyo objeto de estudio son los docentes en activo. En cuanto a los primeros, se ha comprobado que sus creencias condicionan sus aprendizajes, así como el concepto de buen docente que opera en sus prácticas posteriores (Schmidt, 1998). Por ello, se han investigado sus ideas sobre las habilidades y aprendizajes musicales tanto en primaria como en secundaria (Biasutti, 2010); las dificultades que perciben en la función docente, entre las que destacan la falta de experiencia musical, de tiempo y recursos, y el bajo estatus de la asignatura (Russell-Bowie, 2009); las concepciones que presentan sobre la música, su distinción con respecto al ruido y las teorías sobre enseñanza y aprendizaje (Jorquera, 2010); así como las principales causas que atribuyen al fracaso y al éxito en el aprendizaje musical, entre las que subrayan las habilidades y el esfuerzo (Legette, McCord, y Legette, 2016).

Entre los docentes en activo se ha investigado cómo la forma en la que ellos estudiaron música influye en la manera en la que la imparten en la actualidad (Mills \& Smith, 2003); la influencia que tiene su percepción sobre las diferencias individuales de sus estudiantes en los modos de enseñar música (Hewitt, 2005); de qué manera sus percepciones y actitudes con respecto a la improvisación condicionan el valor que le otorgan a esta como herramienta para el desarrollo de la participación y la interpretación en el aula (Koutsoupidou, 2005); o en qué medida el concepto de educación musical del docente y la secuencia didáctica utilizada influyen en la identidad musical que desarrollan los estudiantes (Kelly-McHale, 2013). No obstante, es 
necesario tomar conciencia de la distancia que puede existir entre lo que los docentes declaran y lo que sucede realmente en las aulas (Kennedy y Kennedy, 1996).

Respecto a la pedagogía crítica, si bien esta resulta una realidad poliédrica, aquí se va a acotar a la tradición didáctica que hunde sus raíces en la teoría crítica de la sociedad elaborada por las dos últimas generaciones de la Escuela de Frankfurt. La primera de ellas está representada por la figura de Jürgen Habermas, quien defiende el acto comunicativo como instrumento con potencial crítico sobre la racionalidad instrumental y transformador de la propia realidad (Carbonell, 2015). Su gran aportación a la teoría educativa es lo que se conoce como el aprendizaje dialógico (Aubert, Duque, Fisas, y Valls, 2010; Prieto y Duque, 2009), que se basa en la interacción comunitaria y en la generación de acuerdos intersubjetivos a partir de un diálogo igualitario que respete la inteligencia cultural de todos los individuos (Álvarez, González, y Larrinaga, 2013).

Por su parte, Axel Honneth es el principal representante de la tercera generación y plantea un orden en el que cada individuo sea capaz de desarrollarse como ser autónomo al sentirse reconocido en tres esferas sociales básicas: el amor, el derecho y la solidaridad. Estas confieren al ser humano, respectivamente, autoconfianza, autorrespeto y autoestima (Herzog, 2013). En su aplicación pedagógica, Díaz (2011) sostiene el carácter educativo del proceso de reconocimiento recíproco mediante el que los sujetos, en tanto que ciudadanos, construyen su propia identidad, así como determinadas formas morales de vida. Una de las propuestas didácticas que sigue esta línea es la del reconocimiento de la voz del alumnado en el diseño, gestión o evaluación de la vida escolar (Susinos, 2012), que encuentra en la construcción participativa del currículum (Martínez, 2010) uno de sus máximos exponentes.

En cuanto a las aportaciones de la musicología al concepto de música, la investigación se apoya tanto en la crítica al paradigma formalista de la música autónoma como en el giro culturalista que propició la etnomusicología. Respecto al primero, Wolff (1996) denuncia su hegemonía a finales del siglo XX y sostiene que la música, en tanto que fenómeno cultural, constituye un producto social $\mathrm{y}$, por tanto, no está libre de condicionamientos estructurales. En este sentido, Goehr (1994) llama la atención sobre la forma en la que solemos percibir las obras musicales como expresiones objetivadas y originales de sus compositores, utilizando como referente a la música occidental, europea y clásica. En la misma línea, Talbot (2002) señala la existencia de un compositorcentrismo musical desde principios del siglo XIX que se fundamenta sobre el binomio compositor-obra.

La asunción de este paradigma fomenta el establecimiento de una jerarquía estética y la creación de repertorios canónicos, que acaban determinando los currículums de educación musical. En el ámbito de la educación general, Green (2009) sostiene que durante la mayor parte del siglo XX se priorizó el repertorio de música clásica y sus características de autonomía, universalidad, perennidad y complejidad y que, a pesar de haberse introducido otros tipos de música, los maestros y profesores siguen aplicando la estética de la autonomía musical a repertorios que no deberían ser analizados bajo esa perspectiva. A la vez, los currículums tienden a consolidar los repertorios canónicos. En esta línea, diversos autores denuncian la función de los conservatorios como instituciones que reproducen la legitimación de un repertorio concreto, generalmente de tradición académica occidental (Kingsbury, 1988; Nettl, 1995; Vasconcelos, 2002; Vicente, 2007; Vicente y Aróstegui, 2003).

Con la aparición de la etnomusicología a mediados del siglo XX, comenzó a cobrar valor una concepción culturalista de la música. Entre sus implicaciones actua- 
les, Martí (2000) señala la crisis de una definición universal de música, la necesidad de un juicio emic, la aceptación de dimensiones musicales que trascienden al placer estético y la superación de una historia universal, lineal y progresiva de la música cuyo cénit es la música clásica y europea. Desde este enfoque, gana fuerza la incorporación al currículum de una mayor diversidad de manifestaciones musicales, como la música popular urbana, la no occidental o la tradicional.

Respecto a la primera, Frith (1996) sostiene que representa un espacio cultural de enorme importancia en el que los jóvenes construyen su propia identidad. En relación con este tipo de música, Flores (2007) defiende no solo es un recurso motivador, sino que permite desarrollar muchas destrezas específicas como la improvisación o el desarrollo auditivo dentro de un contexto de aprendizaje informal (Green, 2005). Siguiendo con Martí (2000), el trabajo didáctico con las músicas no occidentales aporta un triple beneficio: ayuda a conocer mejor los fundamentos de la música de tradición europea, añade un componente sociohistórico al fenómeno musical y puede ser motivador para los estudiantes. Por su parte, varios autores (Epelde, 2011; Martín, 2010; Ortiz, 2011; Sánchez, 2014) defienden la necesidad de integrar las músicas no occidentales en el currículo como elemento clave de una política global para la educación intercultural.

Finalmente, algunos autores argumentan la necesidad de trabajar la música tradicional en la educación con el objetivo de conservar un patrimonio musical identitario que se está perdiendo como consecuencia de los procesos de globalización (Arévalo, 2009). Sin embargo, existe todavía poca reflexión sobre la forma descontextualizada en que se aborda este repertorio, dándole en muchos casos un enfoque exclusivamente folklorista. Esta es una postura compartida por Costa (2003), quien asocia la folklorización de tipo ruralista con una ideología conservadora e inmovilista que necesita replantearse los conceptos de música culta, tradicional y popular.

\section{Metodología}

En esta primera fase de la investigación, se ha utilizado como instrumento de análisis un cuestionario formado por 31 ítems construidos a partir de las aportaciones de la musicología y los principios de la pedagogía crítica expuestos, y organizados en torno a tres bloques. El primero ha servido para caracterizar a la muestra en cuestiones como el sexo, la edad, los años de experiencia docente o la utilización de libro de texto. El segundo contenía ítems sobre el repertorio musical utilizado y posibles razones de su selección, como el concepto de música subyacente o los criterios de apreciación estética. En el tercero, se preguntaba sobre el grado de acuerdo con algunos principios del enfoque sociocrítico, así como su nivel de aplicación en el aula. Este instrumento de medición ha sido sometido a un proceso de validación que ha combinado juicio de expertos y prueba piloto.

La población a la que se ha dirigido está constituida por el conjunto de los maestros de música de $5^{\circ}$ y $6^{\circ}$ de Educación Primaria que trabajan en centros de gestión pública del municipio de Valencia ( $\mathrm{N}=92)$, consiguiendo una muestra de un $86,95 \%$ $(\mathrm{n}=80)$. Por tanto, para un nivel de confianza del $95 \%(\mathrm{~K}=1,96)$ se ha obtenido un error muestral del 4\%. Para el análisis de los datos obtenidos, estos se han volcado en el paquete estadístico SPSS, en su versión 23, utilizando descripciones estadísticas, análisis bivariables mediante tablas de contingencia, análisis categoriales y representaciones gráficas. 


\section{Resultados}

A continuación, se presentan los resultados más relevantes, agrupados en torno a los 3 bloques establecidos. Más adelante se analizan algunas relaciones entre variables de distintos bloques.

\subsection{Bloque 1. Caracterización de la muestra}

De manera sintética, la muestra se caracteriza por estar formada por un $68,75 \%$ de mujeres frente a un $28,75 \%$ de hombres. La edad media se sitúa en los 43,39 años, mientras que la media de la experiencia docente lo hace en los 15,56. En cuanto a su formación musical, un $17,5 \%$ ha cursado Grado Superior, un 52,5\% Grado Medio y un 12,5\% Grado Elemental, siendo solo el 15\% quienes no tienes estudios específicos de música. Finalmente, un 38,75\% afirma utilizar libro de texto en sus clases, mientras que un 27,5 afirma no hacerlo y un $30 \%$ hacerlo a veces.

\subsection{Bloque 2. Repertorio musical}

\subsubsection{Porcentaje de uso de cada uno de los repertorios}

La música clásica y romántica ocupa un 22,93\% del repertorio utilizado, seguida muy de cerca por la música escolar $(22,19 \%)$. A continuación, se sitúan la música folklórica y tradicional $(19,72 \%)$, la popular urbana $(16,11 \%)$, la antigua $(9,97 \%)$ y la contemporánea $(5,46 \%)$. De forma minoritaria $(3,62 \%)$ aparece otro tipo de repertorio en el que destacan las bandas sonoras, la música intercultural y las composiciones propias.

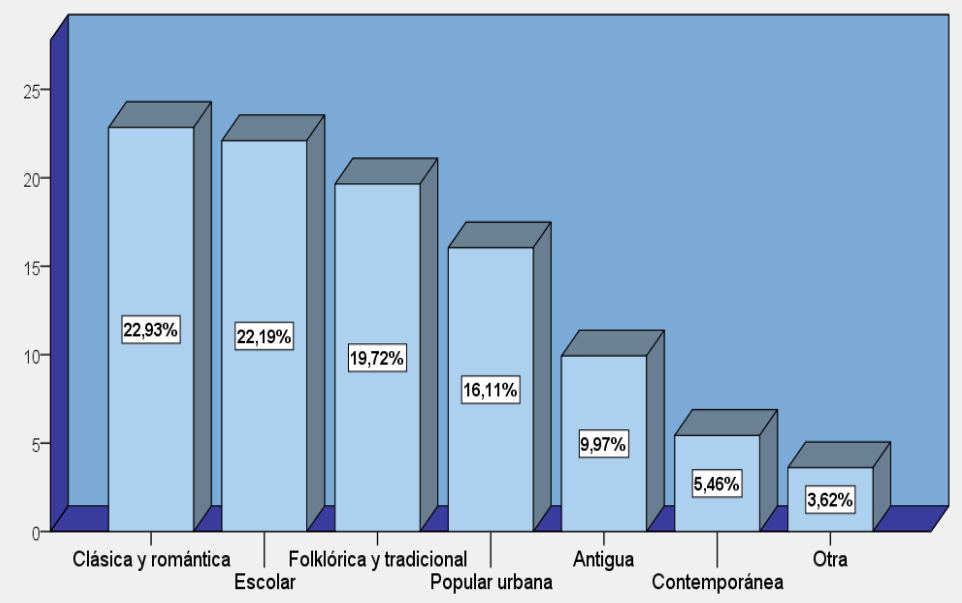

Figura 1. Porcentaje de utilización de cada uno de los repertorios 


\subsubsection{Preferencia a la hora de priorizar un tipo de repertorio}

Un 48,75\% de los encuestados señaló que prefiere priorizar piezas próximas a sus estudiantes y contextos, frente a un $33,75 \%$ que prefiere las grandes obras de la historia de la música. Por otra parte, un 13,75\% marcó ambas opciones.

\subsubsection{Utilización del libro de texto para seleccionar el repertorio}

E1 12,5\% afirma utilizar el libro de texto para seleccionar el repertorio, frente a un $35 \%$ que señala no hacerlo. Por otra parte, un poco más de la mitad $(51,25 \%)$ indica que solo lo utiliza a veces.

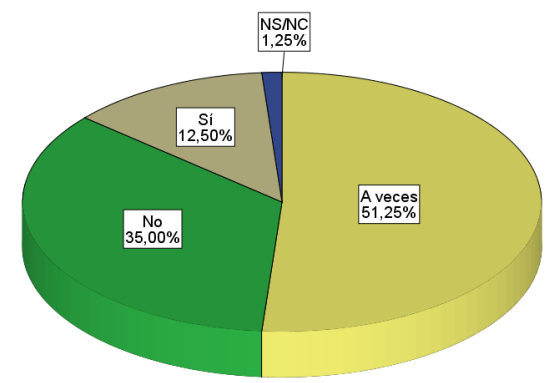

Figura 2. Preferencia de repertorio

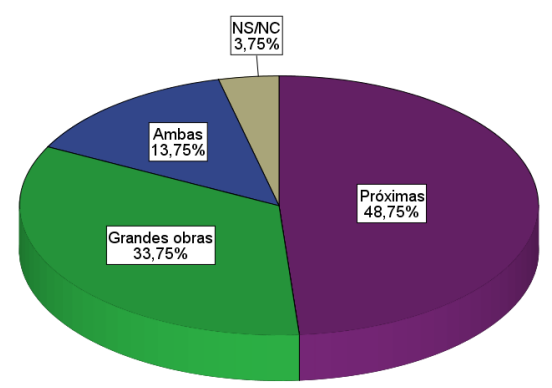

Figura 3. Utilización del libro de texto para seleccionar el repertorio

\subsubsection{Criterios de apreciación estética}

Se ha utilizado una escala Likert de 7 puntos, donde 1 es totalmente en desacuerdo y 7 totalmente de acuerdo. Las 4 afirmaciones formuladas han obtenido puntuaciones similares. En primer lugar se sitúa la valoración de las grandes obras de la historia de 
la música como parte del patrimonio histórico y cultural con una media de 6 , seguida de la idea de que dicho repertorio es el que contiene los elementos fundamentales de la música en su máximo nivel de expresión estética, con una media de 5,47. En tercer y cuarto lugar aparece el repertorio de piezas próximas a los estudiantes, obteniendo un 5,44 en su potencialidad para trabajar la reflexión crítica y un 5,14 en su capacidad para trabajar los elementos fundamentales de la música.

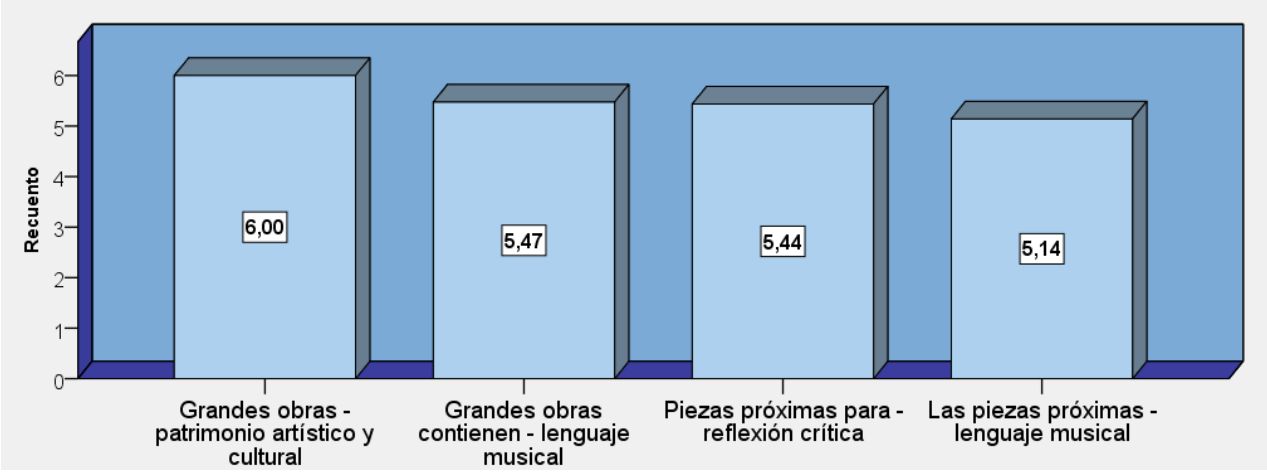

Figura 4. Criterios de apreciación estética

\subsubsection{Concepto de música}

Un 47,5\% de los encuestados declara que su concepto de música está más próximo a entenderla como un fenómeno social vinculado a un contexto cultural determinado, mientras que un $25 \%$ la entiende como un una forma o estructura sonora con criterios de composición e interpretación universales. Por otra parte, un $23,75 \%$ se identifica con ambos conceptos, mientras que un 3,75\% apunta otros, entre los que destaca su relación con las emociones.

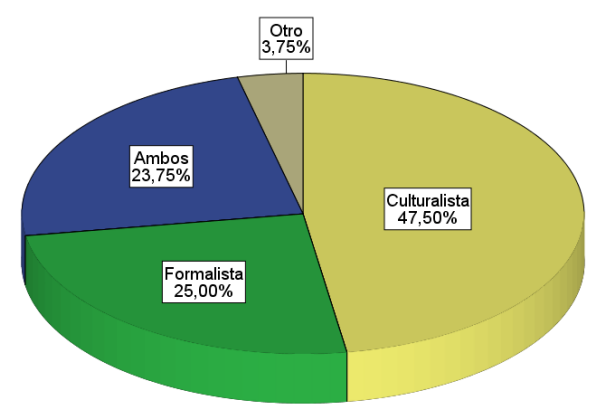

Figura 5. Concepto de música 


\subsubsection{Objetivos de la educación musical}

En este ítem se les pedía a los encuestados que ordenasen los objetivos que aparecen en la figura 6 en orden de importancia, de forma que el 1 fuera el más importante y el 6 el menos importante. El objetivo prioritario es desarrollo de la escucha activa $(1,53)$, seguido del aprendizaje de elementos básicos sobre notación e interpretación $(2,69)$. En tercer lugar, se encuentra la reflexión crítica de la música en relación con su contexto cultural $(3,3 \%)$, muy cerca del conocimiento de las obras más importantes de la historia de la música $(3,6)$. En quinto lugar se sitúa la adquisición de habilidades de improvisación y composición $(4,15)$ y, finalmente, la formación de futuros músicos profesionales $(5,68)$.

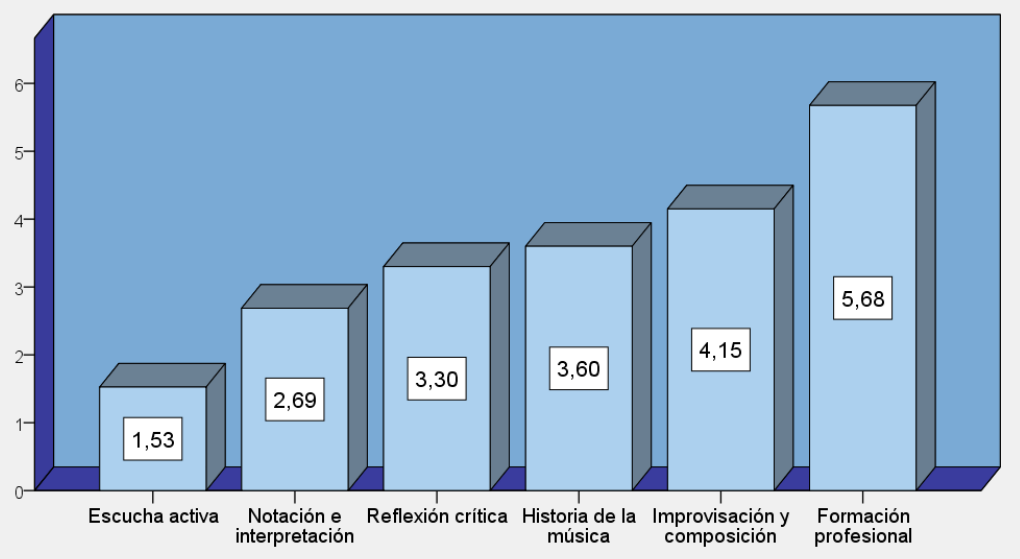

Figura 6. Prioridad de objetivos en educación musical

\subsection{Bloque 3. Enfoque sociocrítico}

\subsubsection{Grado de acuerdo con sus principios y características}

En este caso se pedía valorar mediante una escala Likert de 7 puntos, donde 1 es totalmente en desacuerdo y 7 totalmente de acuerdo, 4 principios o características fundamentales del enfoque sociocrítico. Como se aprecia en la figura 7, entender la educación como una herramienta de transformación social ha obtenido una valoración de 6,5, mientras que introducir un contenido ético, moral y, por tanto, político, ha obtenido una de 5,33. La idea de trabajar de manera interdisciplinar con otras asignaturas se ha puntuado con un 6,27. Por su parte, el valor de tener en cuenta los intereses de los estudiantes en las programaciones ha sido de 5,11. Tanto en este ítem como en el siguiente se han producido unos valores muy elevados debido al sesgo de deseabilidad social. Esto hace que las pequeñas diferencias cobren una mayor importancia a la hora de analizar los resultados 


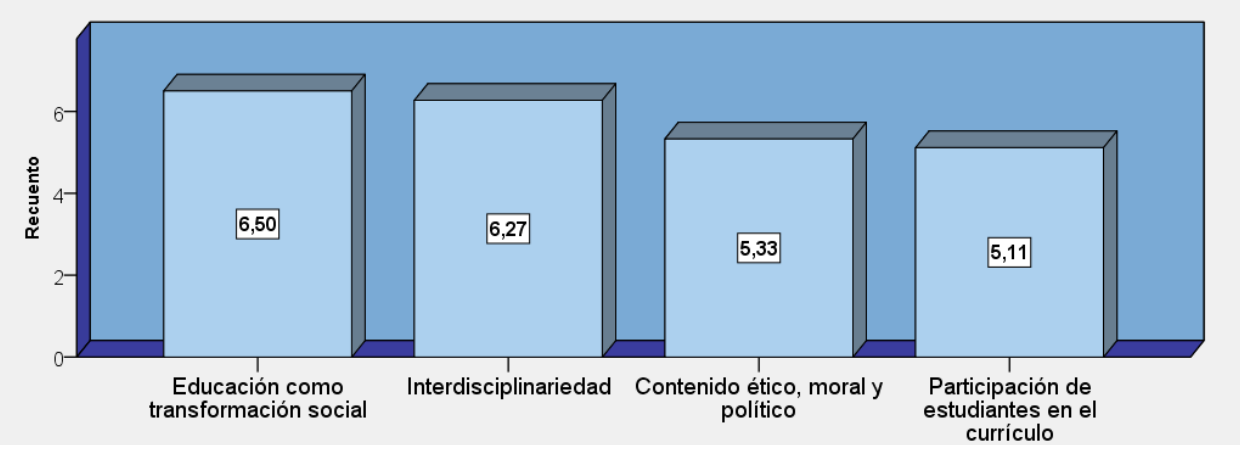

Figura 7. Grado de acuerdo con algunos principios y características del enfoque sociocrítico

\subsubsection{Grado de acuerdo con algunas aplicaciones}

El sistema de valoración de estas afirmaciones fue el mismo que en el punto anterior. Así, la idea de reflexionar de manera crítica sobre las letras de las canciones ha obtenido 5,96 puntos, la de reflexionar sobre el papel de la música en la reproducción de las relaciones sociales 5,81 y la de hacerlo sobre la influencia de la industria cultural en la conformación del gusto musical 5,61.

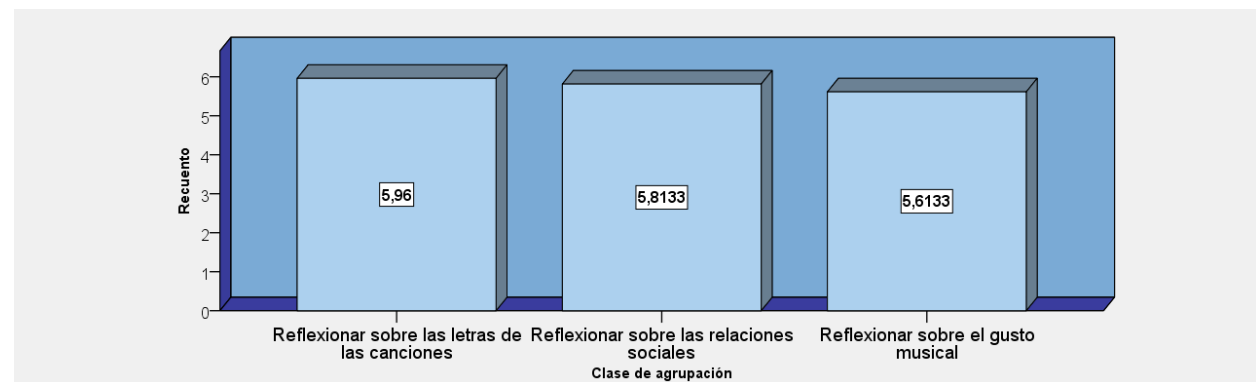

Figura 8. Grado de acuerdo con algunas aplicaciones del enfoque sociocrítico en la educación musical

\subsubsection{Dificultades que puede encontrarse}

De todas las dificultades señaladas, el poco tiempo disponible para la asignatura (45 minutos semanales) ocupa un $37,5 \%$, seguido de la inmadurez de los estudiantes, con un $16,82 \%$. Empatados en tercer lugar se encuentran el problema de la objetividad y el de considerar la música como una asignatura de poca importancia, ambas dificultades con un $15 \%$ de presencia. Por último, entre las que presentan con una representación menor del 5\% agrupadas bajo la categoría otros, destacan la influencia de la industria musical, el poco apoyo familiar y la falta de formación del profesorado. 


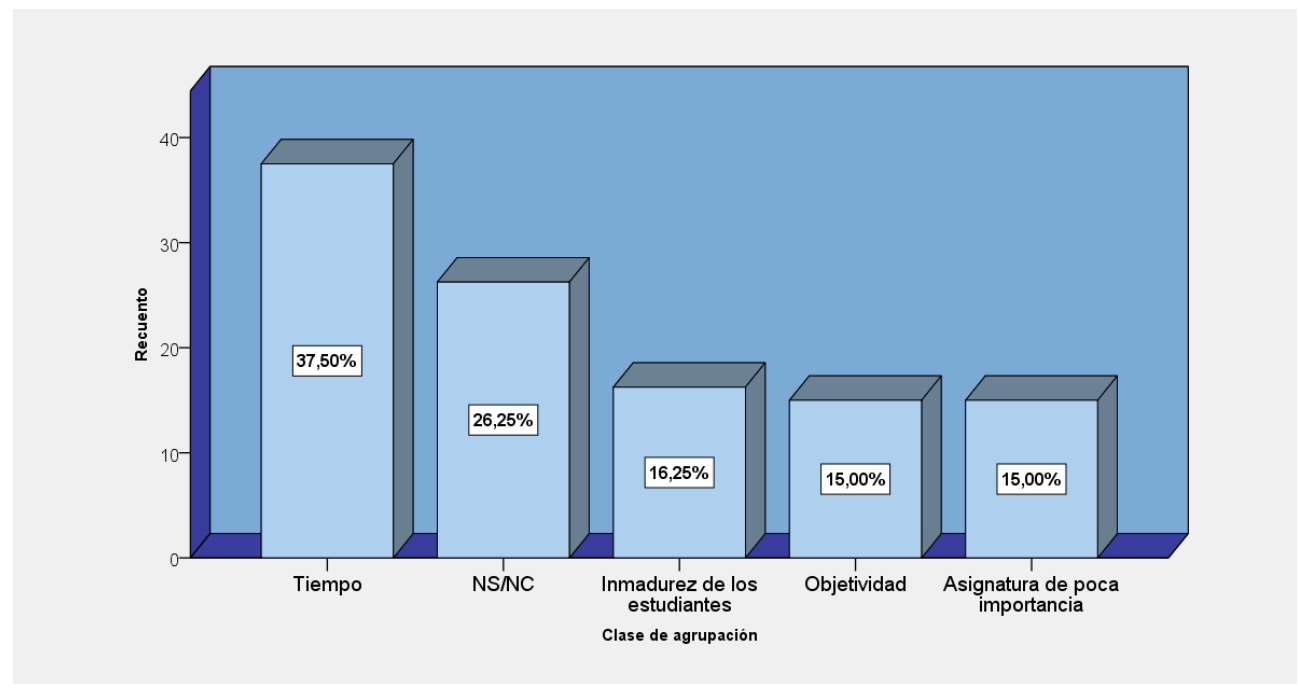

Figura 9. Dificultades de aplicación del enfoque sociocrítico en el aula

\subsubsection{Aplicaciones del enfoque sociocrítico}

Entre todas las respuestas obtenidas, un $60 \%$ no utiliza el enfoque sociocrítico. Entre los usos más frecuentes, aunque con muy poca presencia, se encuentran el debate $(10 \%)$, las reflexiones sobre las relaciones entre música y sociedad $(7,5 \%)$, las actividades en las que los estudiantes tienen que desarrollar un proceso de investigación $(7,5 \%)$ y la incorporación de las preferencias de los estudiantes. Aquellas con una representación inferior al 5\% se han agrupado en otros, destacando la participación de los estudiantes en las programaciones, el análisis crítico de las letras y la utilización de canciones con valores sociales.

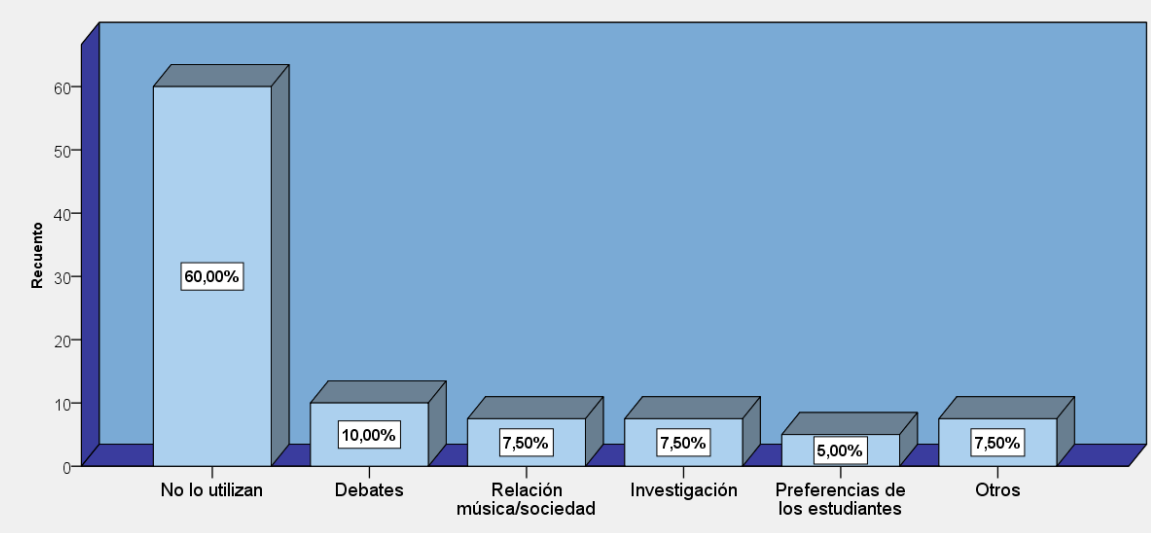

Figura 10. Utilización del enfoque sociocrítico 


\subsection{Relaciones entre variables}

Se han utilizado tablas de contingencia para realizar análisis entre distintas variables. En primer lugar, se ha estudiado la existencia de relaciones entre el concepto de música de los maestros encuestados y el resto de variables, encontrando que influye en las preferencias de repertorio, en el tipo de música más utilizado, en los criterios de apreciación estética estudiados, en los objetivos de la educación musical y en el grado de acuerdo con los principios del enfoque sociocrítico. En segundo lugar, se han triangulado los resultados analizando las relaciones entre todas estas variables dependientes del concepto de música.

Así, como se puede desprender de las tablas 1-4 y de las figuras 11 y 12, aquellos que tiene una concepción de tipo formalista prefieren priorizar las grandes obras de la historia de la música, están más de acuerdo en que estas contienen los elementos del lenguaje musical en su máximo nivel de expresión estética y que constituyen al patrimonio artístico y cultural de la música, utilizan en mayor medida el repertorio clásico y romántico, otorgan una menor importancia a la reflexión crítica y están menos de acuerdo con los principios y características del enfoque sociocrítico. Por su parte, aquellos con una concepción culturalista de la música prefieren priorizar un tipo de repertorio próximo a sus estudiantes, sostienen que dicho repertorio es válido para trabajar los elementos del lenguaje musical y que facilita una reflexión crítica sobre las relaciones entre música y sociedad, utilizan en mayor medida la música folklórica y tradicional, le dan una mayor importancia a la reflexión crítica, y están más de acuerdo con los principios del enfoque sociocrítico.

Tabla 1. Relación de la media de los distintos repertorios con el concepto de música

\begin{tabular}{|c|c|c|c|c|c|c|c|c|}
\hline \multicolumn{2}{|c|}{$\begin{array}{c}\text { Concepto de } \\
\text { música }\end{array}$} & \multirow{2}{*}{$\begin{array}{c}\begin{array}{l}\text { Clásica y } \\
\text { romántica }\end{array} \\
28,36 \\
\end{array}$} & \multirow{2}{*}{$\begin{array}{c}\begin{array}{c}\text { Folklórica y } \\
\text { tradicional }\end{array} \\
16,28 \\
\end{array}$} & \multirow{2}{*}{$\begin{array}{c}\text { Escolar } \\
23,36\end{array}$} & \multirow{2}{*}{$\begin{array}{c}\begin{array}{c}\text { Popular } \\
\text { urbana }\end{array} \\
15,72\end{array}$} & \multirow{2}{*}{$\begin{array}{c}\text { Antigua } \\
7,94\end{array}$} & \multirow{2}{*}{$\begin{array}{c}\text { Contemporánea } \\
\mathbf{6 , 4 0} \\
\end{array}$} & \multirow{2}{*}{$\begin{array}{r}\text { Otra } \\
3,89\end{array}$} \\
\hline Formalista & Media & & & & & & & \\
\hline Culturalista & Media & 19,51 & 20,75 & 23,02 & 16,40 & 9,53 & 6,90 & 3,18 \\
\hline Ambos & Media & 25,63 & 21,32 & 18,11 & 16,16 & 11,42 & 3,68 & 3,68 \\
\hline Otro & Media & 23,33 & 18,33 & 20,00 & 10,33 & 18,00 & 3,33 & 6,67 \\
\hline Total & Media & 23,37 & 19,66 & 21,79 & 15,94 & 9,93 & 5,86 & 3,61 \\
\hline
\end{tabular}

Tabla 2. Relación entre el concepto de música y los criterios de apreciación estética

Informe

\begin{tabular}{|c|c|c|c|c|}
\hline $\begin{array}{c}\text { Media } \\
\text { música }\end{array}$ & $\begin{array}{c}\text { Grandes obras contienen } \\
\text { los elementos del } \\
\text { lenguaje musical en su } \\
\text { mayor nivel de expresión } \\
\text { estética }\end{array}$ & $\begin{array}{c}\text { Las piezas próximas } \\
\text { son igual de válidas } \\
\text { para trabajar los } \\
\text { elementos del } \\
\text { lenguaje musical }\end{array}$ & $\begin{array}{c}\text { Grandes obras } \\
\text { como } \\
\text { patrimonio } \\
\text { artístico y } \\
\text { cultural }\end{array}$ & $\begin{array}{c}\text { Piezas } \\
\text { próximas para } \\
\text { facilitar una } \\
\text { reflexión crítica }\end{array}$ \\
\hline Formalista & $\mathbf{5 , 7 5}$ & $\mathbf{4 , 4 0}$ & $\mathbf{6 , 2 0}$ & $\mathbf{4 , 7 4}$ \\
\hline Culturalista & $\mathbf{5 , 1 6}$ & $\mathbf{5 , 5 4}$ & $\mathbf{5 , 6 8}$ & $\mathbf{5 , 7 3}$ \\
\hline Ambos & 5,74 & 5,26 & 6,42 & 5,53 \\
Otro & 6,33 & 5,00 & 6,33 & 5,67 \\
Total & 5,49 & 5,16 & 6,01 & 5,44 \\
\hline
\end{tabular}


Tabla 3. Tabla de contingencias entre el concepto de música y la preferencia de repertorio

Recuento

\begin{tabular}{|cc|c|c|c|c|c|}
\hline \multirow{2}{*}{} & & \multicolumn{3}{|c|}{ Preferencia en el repertorio } & \multirow{2}{*}{ Total } \\
\cline { 3 - 6 } & $\begin{array}{c}\text { Grandes } \\
\text { obras }\end{array}$ & Próximas & Ambas & NS/NC & \\
\hline \multirow{2}{*}{$\begin{array}{c}\text { Cormalista } \\
\text { música }\end{array}$} & $\mathbf{9}$ & $\mathbf{7}$ & 4 & 0 & 20 \\
& Ambos & 8 & $\mathbf{2 3}$ & 5 & 2 & 38 \\
Total & Otro & 2 & 1 & 2 & 1 & 19 \\
\end{tabular}

Tabla 4. Relación entre el concepto de música y el grado de acuerdo con el enfoque sociocrítico

Media
\begin{tabular}{|c|c|c|c|c|c|c|c|}
\hline $\begin{array}{c}\text { Concepto } \\
\text { de música }\end{array}$ & $\begin{array}{c}\text { Educación } \\
\text { como } \\
\text { transforma } \\
\text { ción social }\end{array}$ & $\begin{array}{c}\text { Contenido } \\
\text { ético, } \\
\text { moraly } \\
\text { político }\end{array}$ & $\begin{array}{l}\text { Interdiscip } \\
\text { linariedad }\end{array}$ & $\begin{array}{c}\text { Participación } \\
\text { de los } \\
\text { estudiantes } \\
\text { en el } \\
\text { currículo }\end{array}$ & $\begin{array}{c}\text { Reflexionar } \\
\text { sobre las } \\
\text { relaciones } \\
\text { sociales }\end{array}$ & $\begin{array}{c}\text { Reflexionar } \\
\text { sobre las } \\
\text { letras de } \\
\text { las } \\
\text { canciones }\end{array}$ & $\begin{array}{c}\text { Reflexionar } \\
\text { sobre el } \\
\text { gusto } \\
\text { musical }\end{array}$ \\
\hline Formalista & $\mathbf{6 , 1 6}$ & $\mathbf{5 , 1 1}$ & $\mathbf{5 , 9 5}$ & $\mathbf{4 , 5 5}$ & $\mathbf{5 , 5 8}$ & $\mathbf{5 , 5 0}$ & $\mathbf{5 , 0 0}$ \\
Culturalista & $\mathbf{6 , 3 5}$ & $\mathbf{5 , 1 2}$ & $\mathbf{6 , 2 9}$ & $\mathbf{5 , 4 2}$ & $\mathbf{6 , 1 4}$ & $\mathbf{6 , 1 8}$ & $\mathbf{5 , 8 7}$ \\
Ambos & 6,47 & 5,53 & 6,32 & 5,00 & 5,28 & 5,74 & 5,47 \\
Otro & 6,00 & 6,00 & 7,00 & 5,00 & 6,67 & 6,67 & 6,33 \\
\hline Total & 6,32 & 5,25 & 6,24 & 5,09 & 5,81 & 5,93 & 5,58 \\
\hline
\end{tabular}

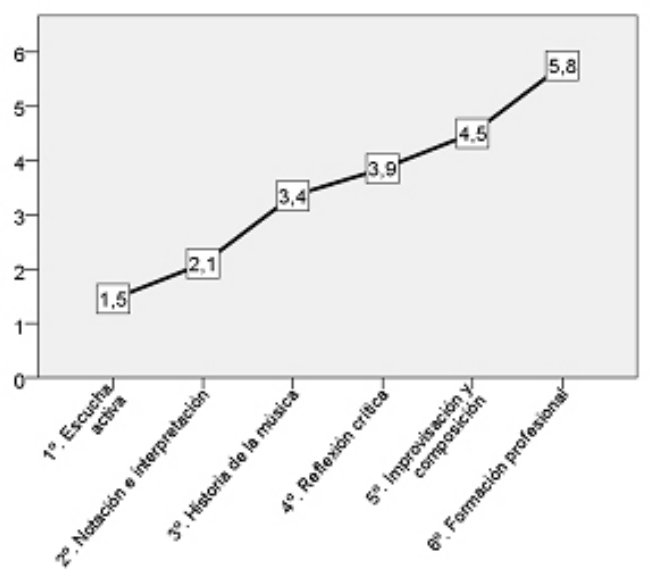

Figura 11. Relación entre el concepto formalista de música y los objetivos educativo

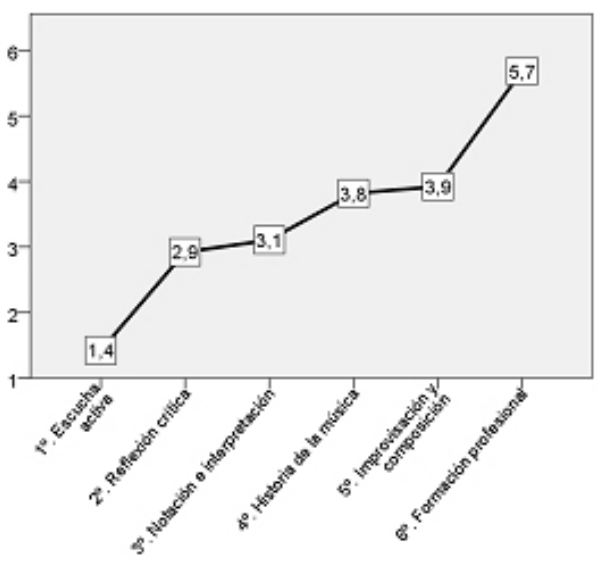

Figura 12. Relación entre el concepto culturalista de música y los objetivos educativos 
Respecto a las relaciones entre las variables en las que influye el concepto de música de los encuestados, se han obtenido los siguientes resultados (tablas 5 y 6 y figuras 13-16). Por un lado, aquellos que prefieren priorizar las grandes obras de la historia de la música sostienen que estas contienen los elementos del lenguaje musical en su máximo nivel de expresión estética y son imprescindibles porque representan el patrimonio artístico y cultural. Además, estos utilizan en mayor medida la música clásica, romántica y antigua. Por su parte, aquellos que prefieren priorizar un repertorio próximo a sus estudiantes sostienen que este es válido para trabajar los elementos del lenguaje musical y que facilita la reflexión crítica. Del mismo modo, utilizan con mayor frecuencia música folklórica y tradicional y música popular urbana. También se ha encontrado que aquellos que dan más valor a las cualidades por las que las grandes obras de la música presentan los elementos del lenguaje musical en su mayor nivel de expresión estética o constituyen el patrimonio artístico y cultural, utilizan con mayor frecuencia la música clásica y romántica. Por otro lado, aquellos que puntúan mejor las cualidades por las que las piezas próximas a los estudiantes y a sus contextos presentan con igual validez los elementos fundamentales de la música o facilitan la reflexión crítica, utilizan con mayor frecuencia la música popular urbana.

Tabla 5. Relación entre la preferencia en el repertorio y los criterios de apreciación estética

\begin{tabular}{|c|c|c|c|c|}
\hline $\begin{array}{c}\text { Preferencia en el } \\
\text { repertorio }\end{array}$ & $\begin{array}{c}\text { Grandes obras } \\
\text { contienen los } \\
\text { elementos del } \\
\text { lenguaje musical en } \\
\text { sumayor nivel de } \\
\text { expresión estética }\end{array}$ & $\begin{array}{c}\text { Las piezas próximas } \\
\text { son igual de válidas } \\
\text { para trabajar los } \\
\text { elementos del } \\
\text { lenguaje musical }\end{array}$ & $\begin{array}{c}\text { Grandes obras } \\
\text { como } \\
\text { patrimonio } \\
\text { artístico y } \\
\text { cultural }\end{array}$ & $\begin{array}{c}\text { Piezas } \\
\text { próximas para } \\
\text { facilitar una } \\
\text { reflexión crítica }\end{array}$ \\
\hline Grandes obras & $\mathbf{6 , 1 9}$ & $\mathbf{4 , 6 7}$ & $\mathbf{6 , 4 8}$ & $\mathbf{5 , 1 1}$ \\
\hline Próximas & $\mathbf{5 , 1 6}$ & $\mathbf{5 , 5 5}$ & $\mathbf{5 , 7 9}$ & $\mathbf{5 , 6 5}$ \\
\hline Ambas & 4,91 & 5,00 & 5,73 & 5,73 \\
NS $N$ N & 5,67 & 5,33 & 5,67 & 4,67 \\
\hline Total & 5,49 & 5,16 & 6,01 & 5,44 \\
\hline
\end{tabular}

Tabla 6. Relación entre la preferencia de repertorio y el porcentaje de uso de cada tipo de repertorio.

Informe

Media

\begin{tabular}{|c|c|c|c|c|c|c|c|}
\hline $\begin{array}{c}\text { Preferencia en el } \\
\text { repertorio }\end{array}$ & $\begin{array}{c}\text { Clásicay } \\
\text { romántica }\end{array}$ & Escolar & $\begin{array}{c}\text { Folklóricay } \\
\text { tradicional }\end{array}$ & $\begin{array}{c}\text { Popular } \\
\text { urbana }\end{array}$ & Antigua & Contemporánea & Otra \\
\hline Grandes obras & $\mathbf{3 1 , 4 8}$ & 20,58 & $\mathbf{1 6 , 4 8}$ & $\mathbf{1 2 , 5 7}$ & $\mathbf{1 3 , 7 4}$ & 4,01 & 2,20 \\
Próximas & $\mathbf{1 9 , 7 1}$ & 22,07 & $\mathbf{2 0 , 7 0}$ & $\mathbf{1 9 , 5 2}$ & $\mathbf{6 , 9 6}$ & 6,56 & 4,59 \\
Ambas & 17,28 & 25,63 & 20,60 & 12,82 & 10,51 & 9,16 & 4,03 \\
NS/NC & 19,00 & 14,67 & 31,67 & 12,33 & 12,33 & 1,67 & 1,67 \\
Total & 23,37 & 21,79 & 19,66 & 15,94 & 9,93 & 5,86 & 3,61 \\
\hline
\end{tabular}




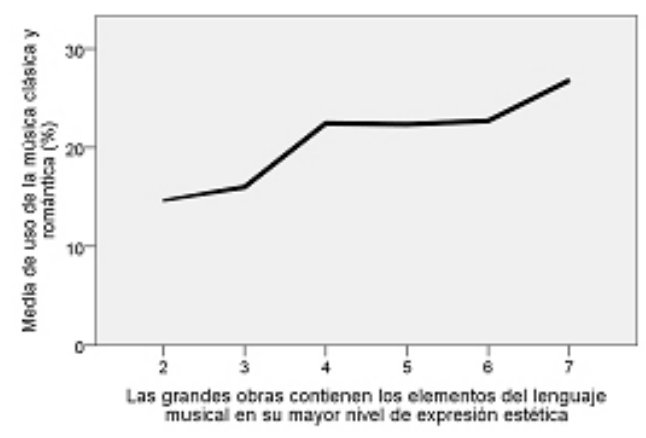

Figura 13. Relación entre criterios de apreciación estética y el uso de la música clásica y romántica (I)

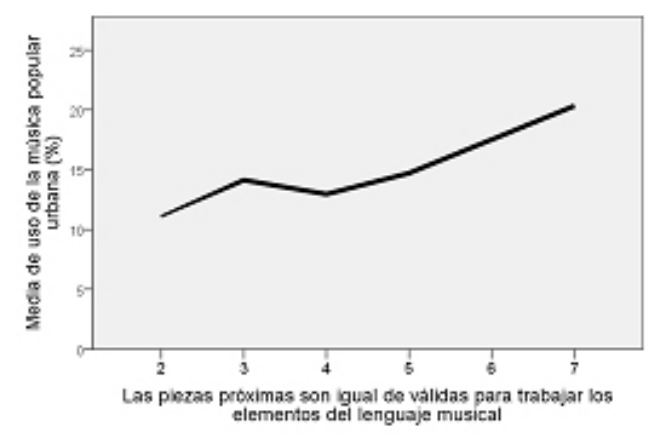

Figura 15. Relación entre criterios de apreciación estética y el uso de la música popular urbana (I)

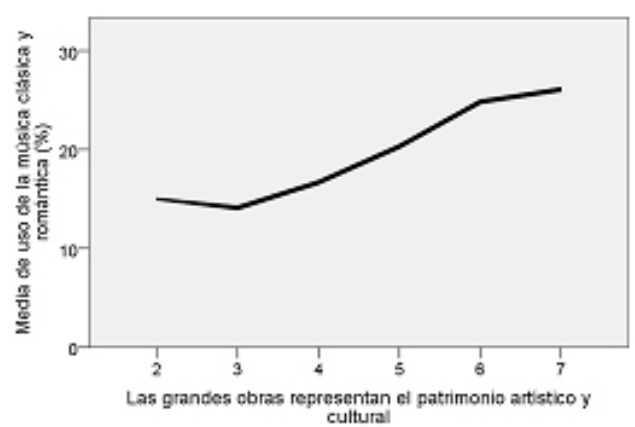

Figura 14. Relación entre criterios de apreciación estética y el uso de la música clásica y romántica (II)

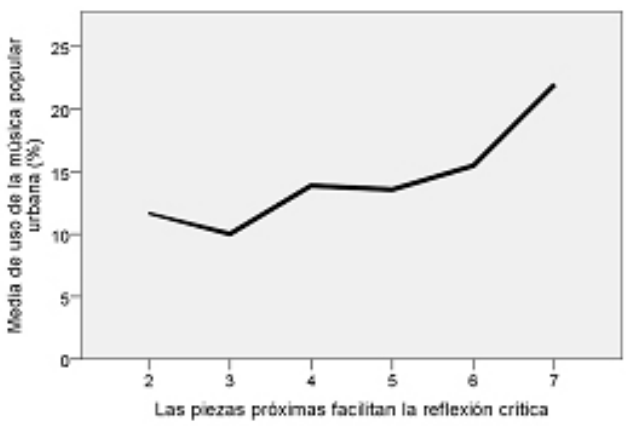

Figura 16. Relación entre criterios de apreciación estética y el uso de la música popular urbana (II)

\section{Conclusiones}

En este estudio se ha realizado un análisis del currículum musical impartido en $5^{\circ}$ y $6^{\circ}$ de Educación Primaria en la ciudad de Valencia, centrado en el repertorio utilizado y la implementación de enfoques de tipo sociocrítico. Los resultados señalan que la música clásica y romántica es la más utilizada, con un $22,93 \%$, seguida de la escolar, con un $22,19 \%$ y de la folklórica y tradicional, con un 19,72\%. Además, si consideramos la antigua, la contemporánea, la clásica y la romántica como parte la música de tradición académica y occidental, esta tiene una presencia del 38,36\%. Por su parte, la música popular urbana ha obtenido un 16,11\%. Los resultados indican que la selección del repertorio está relacionada con el concepto de música que 
tengan los docentes, así como por sus criterios de apreciación estética, como indica el análisis de las relaciones entre variables. Así, se ha comprobado que un concepto de música de tipo formalista, que incluye ideas como la música autónoma (Wolff, 1996), la música absoluta (Dahlhaus, 2006) o la obra de arte musical (Goehr, 1994), se asocia con una mayor utilización de la música clásica y romántica. Por otra parte, aquellos maestros que se han manifestado más afines a un concepto culturalista de la música, entendiendo a esta como un fenómeno asociado a los valores y formas de vida de los distintos grupos humanos en los que se crea, interpreta o recibe, en la línea de las teorías etnomusicológicas (Blacking, 2006; Martí, 2000; Merriam, 1960), utilizan en mayor medida la música folklórica y tradicional.

También se han encontrado correlaciones entre el tipo de repertorio que prefieren priorizar los maestros y el porcentaje de uso de cada uno. En este sentido, se ha visto cómo aquellos que prefieren priorizar las grandes obras de la historia de la música utilizan 1,6 veces más el repertorio clásico y romántico y el doble la música antigua, que aquellos que prefieren priorizar las piezas próximas a los estudiantes. Por su parte, estos últimos utilizan 1,55 veces más la música popular urbana que los primeros, y 1,25 veces más el repertorio folklórico y tradicional.

Otro de los condicionantes a la hora de seleccionar el repertorio son los criterios de apreciación estética. Así, se ha podido demostrar que los maestros que están más de acuerdo en que las grandes obras de la historia de la música contienen los elementos de lenguaje musical en su máximo nivel de expresión artística, o que representan el patrimonio artístico y cultural, tienen una mayor tendencia a utilizar la música clásica y romántica. Por otra parte, aquellos que creen que las piezas próximas a los estudiantes son igual de válidas para trabajar los elementos del lenguaje musical o que facilitan la reflexión crítica sobre las relaciones entre música y sociedad, utilizan con mayor frecuencia la música popular urbana. Por tanto, se ha comprobado que tanto el concepto de música de los maestros, como su preferencia entre las grandes obras de la música y las piezas próximas a sus estudiantes o sus criterios de apreciación estética, condicionan el porcentaje de utilización de algunos de los repertorios planteados. Además, se han encontrado correlaciones entre esos tres elementos que apuntan en la misma dirección. También se ha observado una relación entre el concepto de música y la valoración del enfoque sociocrítico, de forma que aquellos con un concepto culturalista lo valoran más que aquellos con un formalista. Por tanto, a la luz de los resultados, se puede establecer la existencia de dos perfiles de maestros de música, tal y como muestra la tabla 7 . No obstante, no se trata de perfiles rígidos sino de conjuntos de características que muestran una tendencia a presentarse agrupadas.

Respecto al enfoque sociocrítico, se ha producido un alto nivel de acuerdo entre la amplia mayoría de la muestra (5,8 sobre 7$)$, tanto con las características generales como con las concreciones propuestas para el aula de música. Sin embargo, esto no se ve reflejado en la práctica de aula, ya que el 60\% de los maestros manifiesta no utilizarlo, solo un 10\% afirma realizar debates, un 7,5\% declara realizar actividades en las que se trabajan las relaciones entre música y sociedad, y el mismo porcentaje sostiene que utiliza la investigación como metodología de aprendizaje. Entre las razones que aportan los propios encuestados destacan la falta de tiempo, la inmadurez de los estudiantes para realizar reflexiones sociocríticas, la falta de objetividad de este tipo de enfoque o la poca importancia que se otorga a la asignatura. 
Tabla 7. Perfiles de maestros de música.

\begin{tabular}{|c|c|c|}
\hline & Perfil A & Perfil B \\
\hline Concepto de música & Formalista & Culturalista \\
\hline $\begin{array}{c}\text { Preferencia en el } \\
\text { repertorio }\end{array}$ & $\begin{array}{l}\text { Grandes obras de la historia de la } \\
\text { música }\end{array}$ & Piezas próximas a los estudiantes \\
\hline $\begin{array}{l}\text { Criterios de apreciación } \\
\text { estética con mayor } \\
\text { valoración }\end{array}$ & $\begin{array}{l}\text { Las grandes obras de la historia de la } \\
\text { música contienen los elementos del } \\
\text { lenguaje musical en su máximo nivel } \\
\text { de expresión estética y constituyen el } \\
\text { patrimonio artístico y cultural. }\end{array}$ & $\begin{array}{l}\text { Las piezas próximas a los } \\
\text { estudiantes sean igual de válidas } \\
\text { para trabajar los elementos del } \\
\text { lenguaje musical y que faciliten } \\
\text { una reflexión crítica sobre } \\
\text { las relaciones entre música y } \\
\text { sociedad. } \\
\text {. }\end{array}$ \\
\hline $\begin{array}{l}\text { Utilización relativa de } \\
\text { los repertorios }\end{array}$ & $\begin{array}{l}\text { Mayor presencia de la música clásica, } \\
\text { romántica y, puntualmente, antigua. }\end{array}$ & $\begin{array}{l}\text { Mayor presencia de la música } \\
\text { folklórica, tradicional y popular } \\
\text { urbana. }\end{array}$ \\
\hline Enfoque sociocrítico & Menor grado de acuerdo & Mayor grado de acuerdo \\
\hline
\end{tabular}

Como conclusión, se ha podido ver cómo la selección del repertorio musical que se utiliza en el aula está relacionado con las representaciones que tienen los maestros sobre la música. Por tanto, en la medida en que se pretenda incorporar músicas próximas a los estudiantes con el objetivo de implementar en el aula un enfoque didáctico de tipo sociocrítico, la formación del profesorado debería abordar de forma crítica cuestiones propias de la musicología, como las ideas de música autónoma y el giro culturalista de la etnomusicología, así como los principios y concreciones de la pedagogía crítica que contribuyen a la construcción de una ciudadanía democrática, consciente y participativa.

Finalmente, entre las limitaciones de este estudio destaca el hecho de que los datos analizados son declaraciones de los docentes encuestados, de modo que no necesariamente reflejan aquello que pasa en las aulas. No obstante, tanto el repertorio como las concepciones musicales y didácticas pueden ser indicadores fiables de las prácticas educativas. A modo de prospectiva, sería interesante ampliar el estudio a zonas rurales y semiurbanas para conocer si existen diferencias significativas con respecto al contexto urbano. Además, del mismo modo que aquí se ha abordado el currículum impartido, sería conveniente relacionarlo con el prescrito y su concreción en los libros de texto. Igualmente, sería pertinente investigar sobre los gustos musicales de los estudiantes y las narrativas e identidades sociales que estos generan como paso previo a su incorporación al aula de música.

\section{Referencias bibliográficas}

Álvarez, C., González, L. y Larrinaga, A. (2013). Aprendizaje dialógico: una apuesta de centro inclusivo para la inclusión. Tabanque. Revista pedagógica, 26, 209-224.

Arévalo, A. (2009). Importancia del folklore musical como práctica educativa. LEEME, 23, $1-14$

Aubert, A., Duque, E., Fisas, M. y Valls, R. (2010). Dialogar y transformar. Pedagogía crítica del siglo XXI. Barcelona: Graó. 
Biasutti, M. (2010). Investigating trainee music teachers' beliefs on musical abilities and learning: a quantitative study. Music Education Research, 12(1), 47-69.

Blacking, J. (2006). How musical is man? Seattle y London: University of Washington Press.

Cabedo, M. (2014). La música como proyecto socioeducativo. En A. Giráldez (Ed.), Didáctica de la Música en Primaria. Madrid: Editorial Síntesis.

Carbonell, J. (2015). Pedagogías del siglo XXI. Barcelona: Octaedro.

Costa, L. (2003). Práctica pedagógica y música tradicional. LEEME, 12, 1-7.

Cronin-Jones, K. L. (1991). Science teacher beliefs and their influence on curriculum implementation: two case studies. Journal of Research in Science Teaching, 28(3), 235-250.

Dahlhaus, C. (2006). La idea de la Música absoluta. Barcelona: Idea Música.

Díaz, A. (2011). Educación, reconocimiento y ciudadanía. Revista Espaço Pedagógico, 18, $159-270$.

Díaz, M. (2006). Introducción a la investigación en Educación Musical. Madrid: Enclave Creativa.

Epelde, A. (2011). La interculturalidad en la educación a través de la música infantil. DEDiCA. Revista de Educaçao e Humanidades, 1, 273-292.

España. Ley Orgánica 8/2013, de 9 de diciembre, para la Mejora de la Calidad Educativa. Boletín Oficial del Estado, 10 de diciembre, no 295, p. 97858-97921.

España. Real Decreto 126/2014, de 28 de febrero, por el que se establece el currículo básico de la Educación Primaria. Boletín Oficial del Estado, nº 52, p. 19349-19420.

Flores, S. (2007). Principales acercamientos al uso de la música popular actual en la Educación Secundaria. LEEME, 19, 1-16.

Frith, S. (1996). Towards an aesthetic of popular music. En R. Lepperd y S. McClary (Eds.), Music and society. The politics of compositions, performance and reception. New York: Cambridge University Press.

García, F. J. (2010). Música, cultura y sociedad: la enseñanza de la música a partir de problemas socialmente relevantes. Eufonía. Didáctica de la Música, 50, 12-22.

Gil-Pérez, D. y Pessoa de Carvalho, A. M. (2000). Dificultades para la incorporación a la enseñanza de los hallazgos de la investigación e innovación en didáctica de las ciencias. Educación Química, 11(2), 250-257.

Goehr, L. (1994). The imaginary museum of musical works. New York: Oxford University Press.

Green, L. (2005). Meaning, autonomy and autheticity in the music classroom. London: Institute of Education, University of London.

Green, L. (2009). Significado musical y reproducción social: defensa de la recuperación de la autonomía. En D. K. Lines (Ed.), La educación musical para el nuevo milenio. Madrid: Morata.

Herzog, B. (2013). La teoría del reconocimiento como teoría crítica del capitalismo: propuestas para un programa de investigación. Constelaciones: Revista de Teoría Crítica, 5, 311-335.

Hewitt, A. (2005). Teachers' Personal Construct models of pupil individuality and their influence in the music classroom. Music Education Research, 7(3), 305-330.

Jorquera, M. C. (2010). Las ideas de estudiantes de Maestro sobre educación musical. Investigación en la escuela, 70, 101-112.

Kelly-McHale, J. (2013). The influence of music teacher beliefs and practices on the expression of musical identity in an elementary general music classroom. Journal of Research in Music Education, 61(2), 195-216.

Kennedy, C. y Kennedy, J. (1996). Teacher attitudes and change implementation. System, 24(3), 351-360. 
Kingsbury, H. (1988). Music, talent and performance. A conservatory cultural system. Philadelphia: Temple University Press.

Koutsoupidou, T. (2005). Improvisation in the English primary music classroom: teachers' perceptions and practices. Music Education Research, 7(3), 363-381.

Legette, R. M., McCord, D. H. y Legette, L. D. (2016). Causal Beliefs of Pre-service Teachers Regardins Success or Failure in Music Teaching and Learning. Research Perspectives in Music Education, 18(2), 51-59.

Martí, J. (2000). Más allá del arte. La música como generadora de realidades sociales. Sant Cugat de Vallés: Deriva Editorial.

Martín, R. (2010). El papel de la etnomusicología en la educación y en la gestión de las políticas culturales. Historia Actual Online, 23, 85-97.

Martínez, J. (2015). El sujeto político y la educación. Cuadernos de Pedagogía, 455, 91-94.

Martínez, J. B. (2010). El currículum como espacio de participación. La democracia escolar, ¿es posible? En J. G. Sacristán (Ed.), Saberes e incertidumbres sobre el currículum. Madrid: Morata.

Merriam, A. P. (1960). Ethnomusicology: Discussion an Definition of the Field. Etnomusicology, 4(3), 107-114.

Mills, J. y Smith, J. (2003). Teachers' beliefs about effective instrumental teaching in schools and higher education. British Journal of Music Education, 20(1), 5-27.

Nettl, B. (1995). Heartland Excursions. Ethnomusicological Reflections on Schools of Music. Urbana y Chicago: University of Illinois Press.

Ortiz, M. A. (2011). Tendiendo puentes hacia la interculturalidad...desde Andalucía (España) (II). Propuestas desde la Educación Musical. DEDiCA. Revista de Educaçao e Humanidades, 1, 95-116.

Prieto, Ó. y Duque, E. (2009). El aprendizaje dialógico y sus aportaciones a la teoría de la educación. Revista Electrónica Teoría de la Educación. Educación y Cultura en la Sociedad de la Información, 10(3), 7-30.

Russell-Bowie, D. (2009). What me? Teach music to my primary class? Challenges to teaching music in primary schools in five countries. Music Education Research, 11(1), 23-36.

Sánchez, S. (2014). Cultura de Paz y Educación Musical en contextos de Diversidad Cultural. Revista de Paz y Conflictos, 7, 79-97.

Schmidt, M. (1998). Defining «Good» Music Teaching: Four Student Teacher's Beliefs and Practices. Bulletin of the Council for Research in Music Education, 138, 19-46.

Susinos, T. (2012). Las posibilidades de la voz del alumnado para el cambio y la mejora educativa. Revista de Educación, 359, 16-23.

Talbot, M. (2002). The musical work. Teality or invention? New York: Cambridge University Press.

Travé, G. (2003). Música y problemas sociales. Cuadernos de Pedagogía, 328, 48-50.

Vasconcelos, A. A. (2002). O conservatório de música. Professores, organizaçao e políticas. Lisboa: Instituo de Innovaçao Educacional. Ministerio da Educaçao.

Vicente, A. (2007). Evaluación del Currículo en los Conservatorios de Grado Superior de Música en Andalucía. (Tesis doctoral), Universidad de Granada, Granada.

Vicente, A. y Aróstegui, J. L. (2003). Formación Musical y Capacitación Laboral en el Grado Superior de Música, o El Dilema entre los Artístico y lo Profesional en los Conservatorios. LEEME, 12.

Wolff, J. (1996). The ideology of autonomous art. En R. Leppert y S. McClary (Eds.), Music and Society. The Politics of Composition, Performance and Reception. New York: Cambridge University Press. 